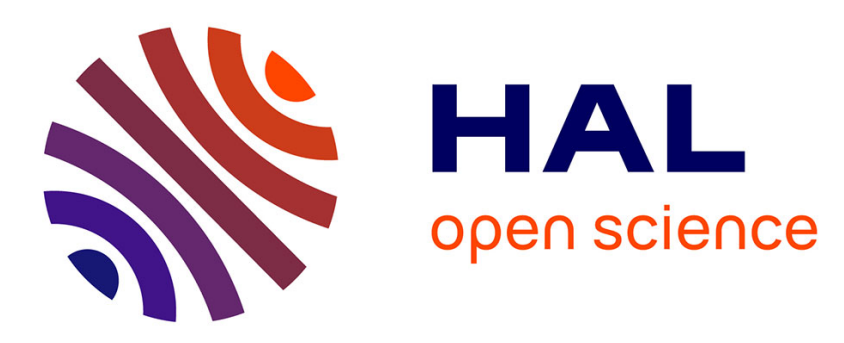

\title{
Nondestructive three-dimensional imaging of crystal strain and rotations in an extended bonded semiconductor heterostructure
}

Anastasios I. Pateras, Marc Allain, Pierre Godard, L. Largeau, G. Patriarche, Anne Talneau, K. Pantzas, M. Burghammer, A. A. Minkevich, Virginie Chamard

\section{To cite this version:}

Anastasios I. Pateras, Marc Allain, Pierre Godard, L. Largeau, G. Patriarche, et al.. Nondestructive three-dimensional imaging of crystal strain and rotations in an extended bonded semiconductor heterostructure. Physical Review B: Condensed Matter and Materials Physics (1998-2015), 2015, 92 (20), pp.205305. 10.1103/PhysRevB.92.205305 . hal-01218363v2

\section{HAL Id: hal-01218363 \\ https://hal.science/hal-01218363v2}

Submitted on 24 Nov 2015

HAL is a multi-disciplinary open access archive for the deposit and dissemination of scientific research documents, whether they are published or not. The documents may come from teaching and research institutions in France or abroad, or from public or private research centers.
L'archive ouverte pluridisciplinaire HAL, est destinée au dépôt et à la diffusion de documents scientifiques de niveau recherche, publiés ou non, émanant des établissements d'enseignement et de recherche français ou étrangers, des laboratoires publics ou privés. 


\title{
Nondestructive three-dimensional imaging of crystal strain and rotations in an extended bonded semiconductor heterostructure
}

\author{
A. I. Pateras, ${ }^{1,2}$ M. Allain, ${ }^{1}$ P. Godard,${ }^{1}$ L. Largeau, ${ }^{3}$ G. Patriarche, ${ }^{3}$ A. Talneau, ${ }^{3}$ K. Pantzas, ${ }^{3}$ M. Burghammer, ${ }^{4}$ \\ A. A. Minkevich, ${ }^{2}$ and V. Chamard ${ }^{1, *}$ \\ ${ }^{1}$ Aix-Marseille Université, CNRS, Centrale Marseille, Institut Fresnel UMR 7249, 13013 Marseille, France \\ ${ }^{2}$ ANKA-Institute for Synchrotron Radiation, Karlsruhe Institute of Technology, 76344 Eggenstein-Leopoldshafen, Germany \\ ${ }^{3}$ Laboratoire de Photonique et de Nanostructures, CNRS, 91460 Marcoussis, France \\ ${ }^{4}$ European Synchrotron Radiation Facility, BP220, 38043 Grenoble Cedex, France
}

(Received 17 June 2015; revised manuscript received 12 September 2015; published 13 November 2015)

\begin{abstract}
We report the three-dimensional (3D) mapping of strain and tilts of crystal planes in an extended InP nanostructured layer bonded onto silicon, measured without sample preparation. Our approach takes advantage of 3D x-ray Bragg ptychography combined with an optimized inversion process. The excellent agreement with the sample nominal structure validates the reconstruction while the evidence of spatial fluctuations hardly observable by other means underlines the specificities of Bragg ptychography.
\end{abstract}

DOI: 10.1103/PhysRevB.92.205305 PACS number(s): 68.37.Yz, 42.30.Rx, 61.05.cp, 61.46.-w

\section{INTRODUCTION}

Imaging the structural properties of a nanostructured crystalline material is a major need of nanoscience, a domain widely driven by the explorations of natural or manufactured crystalline systems, for inspiring new material design routes or understanding new material behavior [1-3]. Answering this need calls for a microscopy method combining sensitivity to the crystalline properties of matter, three-dimensional (3D) imaging capability, in situ compatibility, high spatial resolution, and high sensitivity. In this framework, transmission electron microscopy has largely proven its strength [4] in spite, however, of the invasive sample preparation. Due to this limitation, the recent advent of x-ray lensless imaging methods, based on coherent Bragg diffraction at synchrotrons, opens promising perspectives [5]: to date, X-ray Bragg coherent diffraction imaging [6], holography [7], and ptychography [8-10], performed in situ, have allowed the highly resolved 3D imaging of a large variety of crystals. However, those results are so far restricted to samples with limited dimensions: either of finite 3D size $[6,7,10]$ or extended but along one direction only $[8,9]$. Recently, the more general case of extended films has been considered with Bragg projection ptychography, an efficient approximation of 3D Bragg ptychography, which generates a-still only-2D image of the sample's projected structure factor [11-14]. Bringing x-ray lensless microscopy imaging at a level where its application would represent an asset in nanoscience requires the demonstration of its capacity to provide $3 \mathrm{D}$ imaging in extended nanostructured crystals. Here, we present the 3D mapping of the strain and tilts of crystal planes in an extended layer.

Pushing forward the limit of a newly developed microscopy approach, such as Bragg ptychography, requires the use of a well-calibrated crystalline system, such as the ones arising from the semiconductor bonding technology. This process aims at elaborating silicon-based photonics devices of high quality (e.g., high speed, low power consumption) thanks to the integration of III-V semiconductor heterostructures

\footnotetext{
*virginie.chamard@fresnel.fr
}

onto silicon [15]. The herein employed thin-oxide bonding technology [16] is of particular interest: it falls within the trend of oxide- and metal-free bondings [17], these integration approaches being highly desirable for optimizing the electrical interface quality or the optical index contrast. Consequently, the performances of the designed architecture result not only from the structure of the nominal layer but as well from the bonding quality, including the interface and the integrated layer. From the structural viewpoint, the specificities of the bonding technology strongly calls for a noninvasive exploration of the integrated crystalline architecture, a challenge that Bragg ptychography is expected to address.

Ptychography is an inversion-based microscopy making use of multiple measurements and which combines redundant intensity information from partially overlapping illumination areas [18-20]. 3D x-ray ptychography, originally demonstrated on amorphous materials [21], is based on a combination of 2D ptychography analyses followed by a final tomography reconstruction. The case of crystalline imaging is different. It relies on the $3 \mathrm{D}$ collection and $3 \mathrm{D}$ analysis of a whole set of spatially dependent intensity patterns, measured in the vicinity of a Bragg diffraction peak. It exploits the Bragg sensitivity to crystalline properties, such as strain, lattice rotation, and dislocations. However, measuring extended four- (even five-) dimensional data sets with a focused $\mathrm{x}$-ray beam comes with severe difficulties (stability and positioning accuracy, low signal-to-noise ratio, Bragg intensity level, etc.). Thus, the 3D imaging of a continuous nanostructured crystalline system has not be shown yet. This is the aim of this work.

\section{BRAGG PTYCHOGRAPHY EXPERIMENT}

\section{A. Sample description}

The sample used for the experiment consisted of an InP nanostructured layer, $\langle 001\rangle$ oriented, bonded onto a Si wafer. The InP nanostructured stack was (001) epitaxially grown by metal-organic vapor phase epitaxy with all the layers being lattice matched on an (001) InP substrate. A 300-nm-thick GaInAs sacrificial layer was included in the stack in order to chemically remove the InP substrate by selective etching at the 

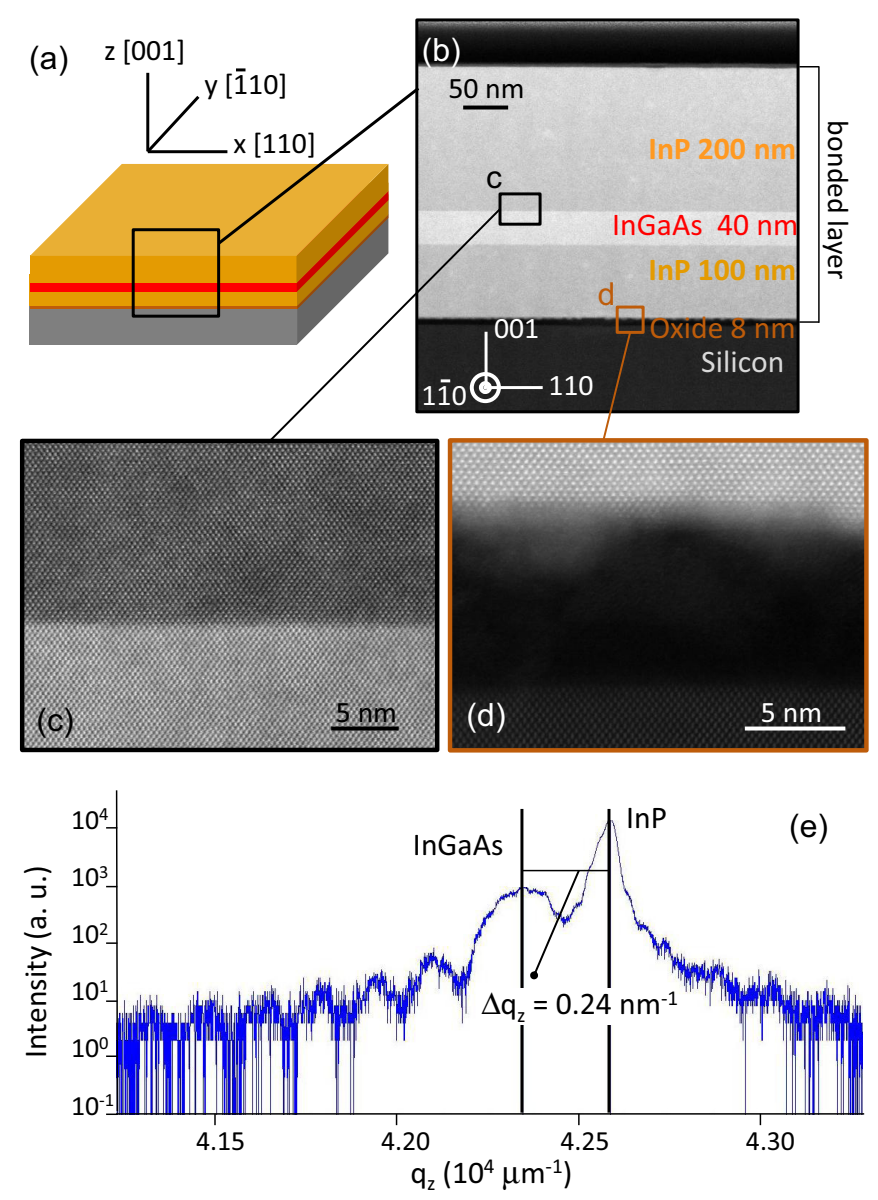

FIG. 1. (Color online) Structure of the InP/Si bonded nanostructured layer. (a) 3D representation of the sample structure. (b) Scanning transmission electron microscopy (STEM) view of the sample cross section (high-angle annular dark field mode). The rectangles indicate zoomed-in regions shown in (c) and (d). (c) Top InP/InGaAs interface and (d) InP/oxide/Si region. (e) High-resolution x-ray diffraction characterization of the InP nanostructured sample performed at the end of the fabrication process, in the vicinity of the $004 \mathrm{InP}$ Bragg reflection.

end of the process. For the integration, a 300- $\mu$ m-thick Si (001) oriented wafer was selected. After cleaning and de-oxidation of both $\mathrm{Si}$ and InP surfaces, both of them were oxidized: the $\mathrm{Si}$ surface was thermally oxidized at $1050{ }^{\circ} \mathrm{C}$ during $20 \mathrm{~s}$ while the InP surface was oxidized during 4 min under an inductive coupled plasma reactive ion etching operated at zero bias. Both oxides were then activated by ozone during $30 \mathrm{~s}$ before being put in contact and annealed at $300^{\circ} \mathrm{C}$ during 3 hours [16]. The InP substrate was then chemically removed, followed by the GaInAs selective layer removal. The whole procedure led to a nanostructured InP stack bonded on $\mathrm{Si}$, schematically shown in Fig. 1(a). The nanostructured stack was composed of a 200-nm-thick InP layer, a 40-nm InGaAs layer, and a 100-nm InP layer, from the surface to the bonding interface.

The details of the crystalline structure were investigated with scanning transmission electron microscopy (STEM), in the same region as the one used for the Bragg ptychography measurement [Fig. 1(b)]. To this aim, the selection of the sample part was done after the synchrotron experimental campaigns. The localized sample cross sections were prepared with a focused ion beam using a FEI SCIOS dual-beam system in order to achieve electron transparency. The ion etching was performed at high voltage around the region of interest, before the as-prepared lamella was lifted out and transferred to a transmission electron microscopy grid for final polishing at low voltage. The high-resolution STEM observations were performed using a JEOL 2200 FS microscope equipped with a probe aberration corrector. This corrector allowed a spatial resolution below $0.1 \mathrm{~nm}$ in this imaging mode. The chemical contrast was obtained by collecting the scattered electrons at very high angle using a high-angle annular dark field (HAADF) detector. In these configurations, heavy elements gave bright contrast. On a local scale, the high quality of the integration process is illustrated in Fig. 1(c), where the top $\mathrm{InP} / \mathrm{InGaAs}$ interface is shown on an area of about $50 \times$ $50 \mathrm{~nm}^{2}$. At the bonding interface [Fig. 1(d)], the 8-nm-thick oxide layer exhibits chemical inhomogeneities with typical length scale of $10 \mathrm{~nm}$. Transmission electron microscopy, which is more sensitive to the presence of extended defects, was also performed and did not reveal the presence of dislocations in this area.

The chosen composition of the embedded layer, $\mathrm{In}_{0.57} \mathrm{Ga}_{0.43}$ As, produced a well-calibrated lattice mismatch, in agreement with the Vegard law. After its fabrication, the InP nanostructured layer was characterized by high-resolution $\mathrm{x}$ ray diffraction with an XPert Pro MRD PANalytical laboratory diffractometer equipped with a $\mathrm{Cu}-\mathrm{K} \alpha$ source. The $q_{z}$ scan $(\theta / 2 \theta$ scan $)$ performed in the vicinity of the InP 004 Bragg reflection and shown in Fig. 1(e) allowed us to evaluate the InGaAs vertical strain to $\Delta a / a=0.56 \%$ with respect to the surrounding InP crystal. Moreover, the presence of well-defined Bragg peaks and thickness fringes demonstrated that the vertical strain in the embedded InGaAs layer was homogeneously preserved through the bonding process. Additional x-ray Bragg diffraction measurements showed that the strain developed mainly along the $\langle 001\rangle$ crystallographic axis.

\section{B. Bragg ptychography experiment}

The Bragg ptychography measurements were made at the ID13 beamline at ESRF (European Synchrotron Radiation Source) with a monochromatic beam of wavelength $\lambda=$ $0.083 \mathrm{~nm}$ (bandwidth $\delta \lambda / \lambda \approx 10^{-4}$ ). The experiment was performed directly on the extended nanostructured layer, without sample preparation.

The finite-sized beam spot produced by the focalization of a fully coherent beam using a set of refractive Si lenses with focal length of about $0.01 \mathrm{~m}$ was characterized in detail prior to the Bragg ptychography measurement. The knowledge of the illumination function is a key parameter in Bragg ptychography, as it governs the choice of the ptychography scanning parameters. Furthermore, the illumination function has to be introduced during the ptychography inversion in a deconvolution operation [8]. Hence, in order to obtain the phase and amplitude of the wave field in the focal plane, a lensless microscopy method was used, inspired by Ref. [22] and described in detail in Refs. [9,10]. It is based on the simple and fast measurement of the overfocused direct beam intensity pattern performed with a high-resolution camera. To this aim, 

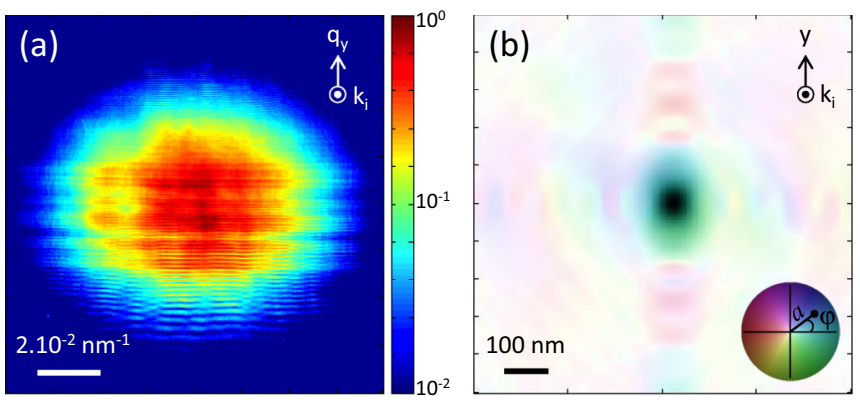

FIG. 2. (Color online) Characterization of the x-ray illumination profile. (a) Coherent intensity pattern of the overfocused beam (arbitrary units) measured with a high-resolution camera. (b) Color rendition of the complex-valued beam profile, retrieved from the inversion of (a) and shown in the plane perpendicular to the incident beam direction, at the sample position. The brightness and color correspond to the beam amplitude $a$ (linear scale) and phase $\varphi$, respectively. Note that $\mathbf{y}$ is along the laboratory vertical direction.

a PCO camera with pixel size of approximately $1.9 \mu \mathrm{m}$ was used, located at a distance of $1.81 \mathrm{~m}$ from the focal plane. This configuration allows a high oversampling of the diffraction pattern [Fig. 2(a)], whose fine structures, such the interference fringes resulting from the Fresnel propagation of the coherent wave front truncated by the slits defining the lens aperture, can clearly be observed (slit aperture set to $60 \times 64 \mu \mathrm{m}^{2}$ in the vertical and horizontal directions, respectively). The measured intensity was inverted with our phasing routine before the beam was back-propagated down to the plane corresponding to the sample position. As expected from the Gaussian absorption profile of the refractive lenses, the beam profile amplitude behaves closely to a Gaussian function with however some weak secondary maxima visible on both horizontal and vertical sides of the central lobe in Fig. 2(b). The widths of the central lobe taken between the first zeros of the amplitude pattern are $240 \mathrm{~nm}$ and $270 \mathrm{~nm}$ along the horizontal and vertical directions, respectively. This beam profile results in an intensity spot (i.e., the squared amplitude) of full width at half maximum of $55 \mathrm{~nm}$ and $60 \mathrm{~nm}$, along the same respective directions.

The InP sample was mounted vertically on a three-axis piezo stage fixed on the top of a hexapod device. The accurate alignment of the center of rotation with regards to the focal plane was ensured by the use of an optical microscope with short depth of field (about $1 \mu \mathrm{m}$ ). The sample was then oriented so that the InP 004 Bragg diffraction conditions were met (Bragg angle $\theta_{B}=16.38^{\circ}$ ). In the following, $\mathbf{G}_{004}$ refers to the InP 004 Bragg reflection, with $\left|\mathbf{G}_{004}\right|=4.258 \times$ $10^{4} \mu \mathrm{m}^{-1}$. The shallow Bragg angle resulted in an elongation of the illumination footprint onto the sample surface, up to $850 \times 270 \mathrm{~nm}^{2}$ along the $\mathbf{x}$ and $\mathbf{y}$ directions, respectively (corresponding to a FWHM spot intensity of $200 \times 60 \mathrm{~nm}^{2}$ [Fig. 3(a)]). The large lattice mismatch between the InP and the Si crystals $(\approx 8 \%$ along $\langle 001\rangle)$ allowed us to isolate the scattering signal arising from the InP nanostructured layer.

The coherently diffracted intensities were collected with a 2D Maxipix detector (pixel width of $55 \mu \mathrm{m}$ ) [23], located $1.38 \mathrm{~m}$ away from the sample [Figs. 3(a), 3(b)]. The whole $5 \mathrm{D}$ data set was obtained by scanning first the beam-to-sample
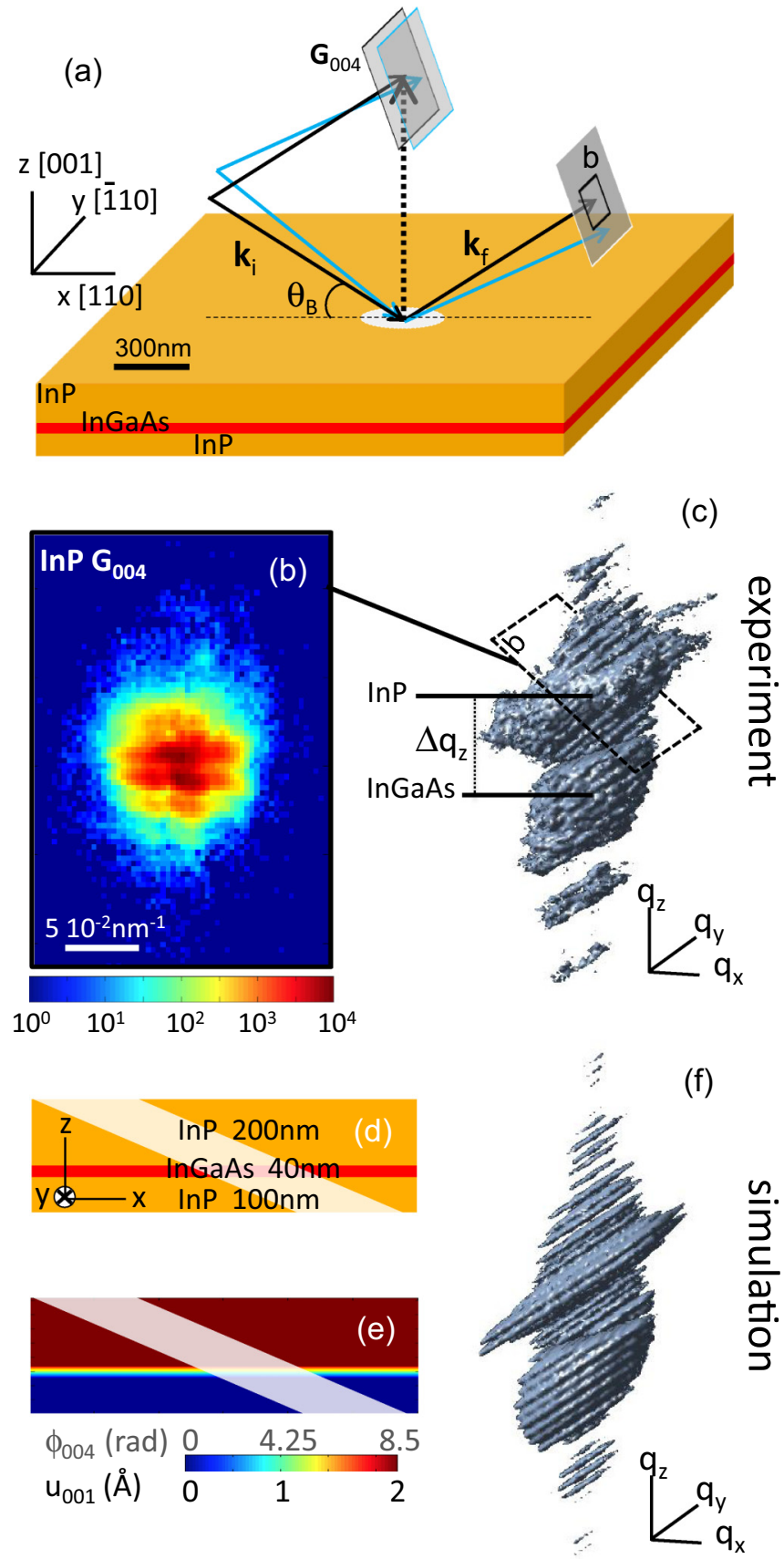

FIG. 3. (Color online) 3D Bragg ptychography acquisition. (a) Schematic of the experiment performed near the InP 004 reflection. The intensity is recorded as a function of the $3 \mathrm{D}$ momentum transfer $\mathbf{q}$ by combining $2 \mathrm{D}$ detector measurements acquired along the rocking curve. Note that $\mathbf{q}=\mathbf{k}_{f}-\mathbf{k}_{i}$, where $\mathbf{k}_{i, f}$ are the incident and exit wave vectors, respectively. The white ellipse shows the footprint of the incident beam FWHM intensity. (b) Zoomed-in region of a $2 \mathrm{D}$ acquisition obtained at the $\operatorname{InP} \mathbf{G}_{004}$ and (c) one 3D experimental intensity pattern (isointensity rendering with threshold at 10 photons). 3D simulations are performed using the nominal values of the InP nanostructured layer: (d) schematic of the density together with the inclined illumination in white, (e) (001) component of the displacement field map and corresponding phase. (f) Simulated 3D pattern [same threshold as (b)]. In (c) and (f), the lengths of the axis represent $1.5 \times 10^{-2}, 1.5 \times 10^{-1}, 1.5 \times 10^{-1} \mathrm{~nm}^{-1}$ along $\mathbf{q}_{x}, \mathbf{q}_{y}, \mathbf{q}_{z}$, respectively. 
position for $11 \times 9$ positions along $\mathbf{x}$ and $\mathbf{y}$, respectively, and repeating this raster scan at each angle along the rocking curve (180 frames with angular step of $\left.0.003^{\circ}\right)$. The needed redundancy in the data set was obtained from a strong overlapping $(\approx 80 \%)$ between successive illumination area, ensured by step sizes of $150 \mathrm{~nm}$ and $50 \mathrm{~nm}$ along $\mathbf{x}$ and $\mathbf{y}$, respectively. The acquisition was performed by repeating 3 times the diffraction pattern measurement with fixed scan parameters (angular and spatial positions), in order to avoid saturation of the detector at the intense InP Bragg peak and increase the signal-to-noise ratio. The total acquisition time was set to $3 \times 0.2 \mathrm{~s}$ per position, leading to a maximum intensity of about $10^{4}$ photons per pixel. A peculiar attention was given to the monitoring of possible radiation damage (monitored over $50 \mathrm{~min}$ ) and beam instability: none of them were observed.

\section{Ptychography data preliminary analysis}

Prior to the inversion, the intensity distribution $I\left(\mathbf{q}, \mathbf{R}_{n}\right)$ was extracted as a function of $\mathbf{q}$, the reciprocal space position, and $\mathbf{R}_{n}$, the $n$th beam-to-sample position. One of these is shown in Fig. 3(c), plotted in the $\left(\mathbf{q}_{x}, \mathbf{q}_{y}, \mathbf{q}_{z}\right)$ reciprocal space frame, the frame conjugated to the laboratory $(\mathbf{x}, \mathbf{y}, \mathbf{z})$ frame. As expected from the shape and finite extent of the scattering volume [i.e., the intersection of the layer and the illumination, Fig. 3(d)] the corresponding intensity distribution presents a three-dimensional extended and inclined shape. The fringes along $\mathbf{q}_{z}$ as well as the InP and InGaAs Bragg peaks, separated as expected by $\Delta q_{z}=\Delta a / a \times\left|\mathbf{G}_{004}\right|=0.24 \mathrm{~nm}^{-1}$, are clearly identified. To quantify further the data quality, the experiments were compared to numerical simulations, using the 3D complex-valued electron density

$$
\rho(\mathbf{r})=|\rho(\mathbf{r})| \exp i \phi(\mathbf{r}),
$$

where $\mathbf{r}$ is the position inside the sample, $|\rho(\mathbf{r})|$ is the density in the ideally unstrained crystal, and $\phi(\mathbf{r})$ is a phase, which holds information on the crystalline properties through the introduction of the crystalline displacement field function $\mathbf{u}(\mathbf{r})$ and the chosen Bragg vector. For $\mathbf{G}_{004}$, one gets [24]

$$
\phi_{004}(\mathbf{r})=\mathbf{G}_{004} \cdot \mathbf{u}(\mathbf{r})=\left|\mathbf{G}_{004}\right| u_{001}(\mathbf{r}),
$$

where $u_{001}(\mathbf{r})$ is the projection of $\mathbf{u}(\mathbf{r})$ onto $\mathbf{G}_{004}$. This formalism allows us to write the averaged expected Bragg intensity $I\left(\mathbf{q}, \mathbf{R}_{n}\right)$ as

$$
I\left(\mathbf{q}, \mathbf{R}_{n}\right)=\left|\mathcal{F}\left(P_{n} \times \rho\right)(\mathbf{q})\right|^{2},
$$

where $\mathcal{F}$ is the Fourier transform and $P_{n}(\mathbf{r}):=P\left(\mathbf{r}-\mathbf{R}_{n}\right)$ is the $3 \mathrm{D}$ complex-valued illumination function at the sample position [8]. In our case, the composition and strain distribution models were defined according to the nominal structure, resulting in the $u_{001}(\mathbf{r})$ and $\phi_{004}(\mathbf{r})$ distributions shown in Fig. 3(e). The main characteristics of the 3D experimental data (inclinations and positions of peaks, fringes) are reproduced by the model [Fig. 3(f)]. However, while the measured InGaAs peak shape agrees well with the simulation, visible discrepancies are observed on the InP peak extent. They necessarily arise from structural distortions, whose presence is confirmed below.

To this aim, a subset of $\left(\mathbf{q}_{x}, \mathbf{q}_{z}\right)$ intensity patterns obtained at successive $\mathbf{R}_{n}$ 's was extracted from the 5D data set [Fig. 4(a)]. The smooth appearances of intensity distortions as a function of $\mathbf{R}_{n}$ are clear signatures of structural inhomogeneities. The

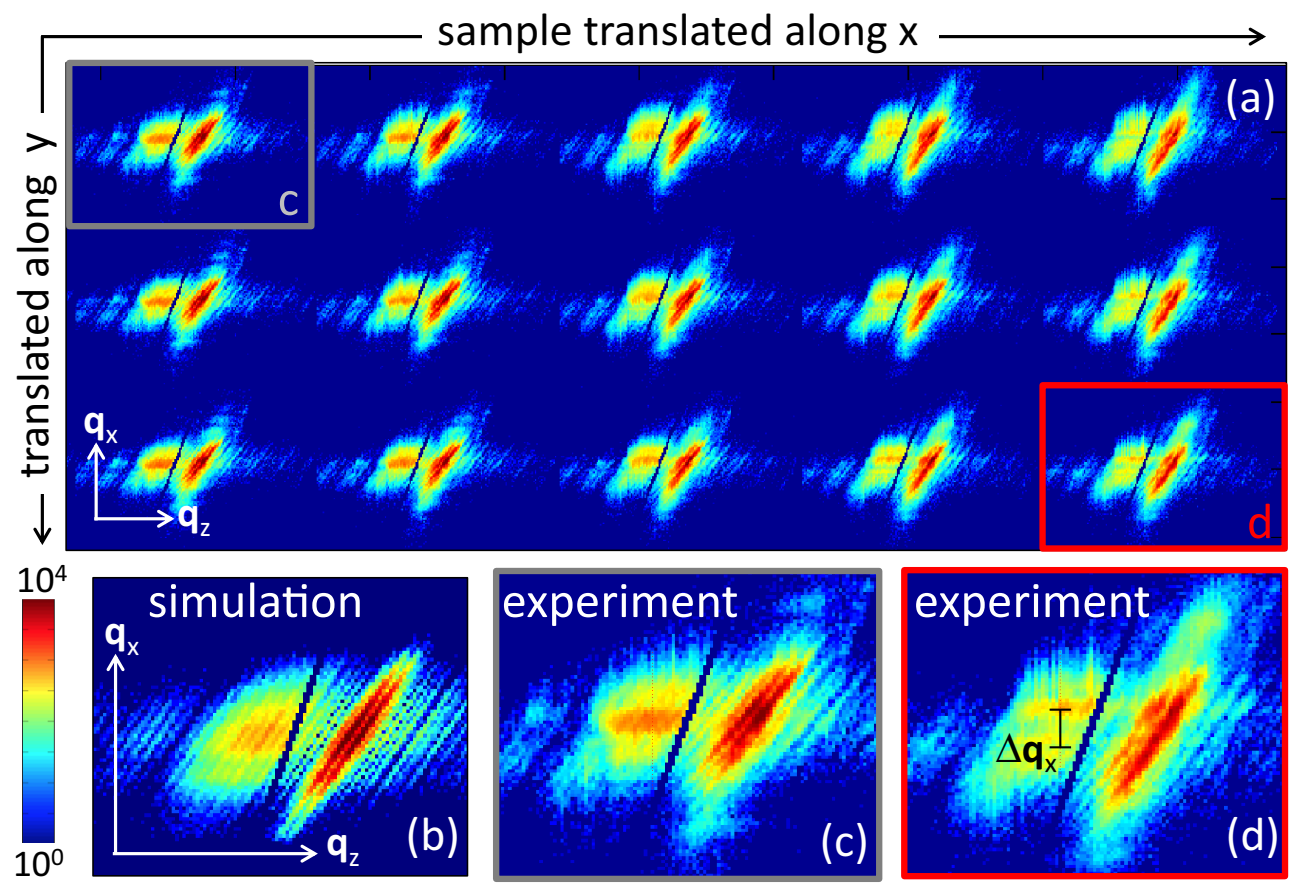

FIG. 4. (Color online) Bragg ptychography data set. (a) Subset of ptychography diffraction patterns $\left[\left(\mathbf{q}_{x}, \mathbf{q}_{z}\right)\right.$ plane $]$ acquired at successive beam-to-sample positions. (b) Same cross section extracted from the 3D simulation of Fig. 3(e). (c), (d) Zoomed-in regions of the two selected intensity patterns shown in (a). The length of the white arrows corresponds to $5 \times 10^{-2}$ and $5 \times 10^{-1} \mathrm{~nm}^{-1}$ for the $\mathbf{q}_{x}$ and $\mathbf{q}_{z}$ directions, respectively. 
comparison between two zoomed-in patterns from different $\mathbf{R}_{n}$ 's with the simulated cross section [Figs. 4(b)-4(d)] allows evidencing the onsets of the structural inhomogeneities. They manifest themselves as a general broadening of the InP peak together with an additional splitting along $\mathbf{q}_{x}$ (with $\Delta q_{x} \approx$ $9.4 \times 10^{-3} \mathrm{~nm}^{-1}$ ), observed on specific sample positions. This latter corresponds to the coexistence of crystals pointing at slightly different directions, where the angular distance, given by $\tan ^{-1}\left(\Delta q_{x} /\left|\mathbf{G}_{004}\right|\right)$, is estimated here to about $0.015^{\circ}$. We will see that this value is consistent with our numerical simulations introducing local crystalline plane tilts (Sec. IV B). Their presence is as well evidenced by the Bragg ptychography reconstruction shown now, which gives additionally a spatial representation of the crystalline properties.

\section{BRAGG PTYCHOGRAPHY 3D RECONSTRUCTION}

The inversion of the Bragg ptychography data set allows us to retrieve the complex-valued effective density, as modeled by Eqs. (1) and (2). The whole set of diffraction patterns (99 positions) was used. In order to keep consistency between the scanning steps and the direct space pixel size, the intensity matrices were shaped to a volume of $542 \times 130 \times 190$ voxels. This results, in the orthogonal direct space, in a voxel of size $\delta x=15.5 \mathrm{~nm}, \delta y=16.5 \mathrm{~nm}$, and $\delta z=9 \mathrm{~nm}$. We note that the use of a 3D Fourier transform to model the far-field intensity directly from the knowledge of the 3D sample and probe [Eq. (3)] implicitly assumes that the probe volume remains constant during the rocking scan. This is in principle not correct as the footprint along the $x$ axis depends on the angle of incidence. However the reduced extent of the angular scan (about $0.5^{\circ}$ ) corresponds to a footprint shrinkage of about $3 \%$ $(\approx 10 \mathrm{~nm})$, slightly smaller than one pixel along $x$. This effect can therefore be neglected. In the future, it will be possible to take it fully into account with the use of the new formalism developed in Ref. [25].

The inversion cycle requires first to define the probe and sample estimates. The 3D sample estimate was calculated from the nominal structural parameters, while the 3D probe was obtained from the 2D complex-valued profile shown in Fig. 2(b) and assumed to be invariant along the propagation direction. This assumption is perfectly valid as the x-ray beam depth of field is 2 orders of magnitude larger than the probed sample thickness [26]. The inversion of the 5D data set was performed with a specifically optimized hybrid strategy based on our phase retrieval algorithms, which are extensively described in Ref. [27]. The gradient-based ordered-subset (OS) algorithm was first used for 1000 iterations. During the first 800 iterations, an amplitude constraint was applied then removed for the last 200 iterations. This first inversion step produced a high-quality estimate, which was further injected into a preconditioned conjugated scaled-gradient (CSG) algorithm for 1000 more iterations. Again the amplitude constraint was applied for the first 500 iterations. During both inversion steps (OS and CSG) a regularization of the searched solution was used [9] penalizing the reconstruction for every pixel outside a finite-thickness planar support (353 nm thick along z). The regularization parameter $\mu$ was set to $10^{7}$. All along the inversion, the Gaussian probability distribution function was introduced for modeling the photon noise statistics. In total, the computing time for the whole inversion was about 117 hours. We observed that the CSG inversion made noticeable refinements on the previously obtained OS reconstruction, pointing out the two main goals of this proposed combination of two algorithms: (i) taking advantage of the good initial convergence properties of the OS, that occurs during the first iterations only, and (ii) avoiding potential stagnation of the CSG at local minima, by initializing it with a proposed solution that already reproduces fairly the experimental data [27].

The resulting 3D amplitude and phase are shown in Figs. 5(a) and 5(b). Although the sample is an extended film, the shown volume presents a finite size corresponding solely to the region which was illuminated during the ptychography scan. The oscillations in the amplitude map are artifacts arising from the use of the regularization approach onto noise-corrupted data, as shown by the preliminary numerical analysis we performed in parallel to this study. They however affect only weakly the phase map, which presents a strong variation at the position of the expected InGaAs layer. For this well calibrated crystalline nanostructured sample, the quality of the reconstruction can be further estimated from the extraction of $\epsilon_{z z}$, the 001 component of the strain map. This latter is obtained from the partial derivative of the phase $\phi_{004}(\mathbf{r})$ with respect to the $z$ coordinate. In Fig. $5(\mathrm{c})$, the obtained 3D $\epsilon_{z z}$ map demonstrates clearly the performances of this microscopy approach: the embedded strained layer is retrieved with position, thickness, and strain mean value corresponding to the nominal structure of the InGaAs layer. From the fluctuations observed in the $1 \mathrm{D} \epsilon_{z z}$ profile [Fig. 5(d)], we estimate the strain sensitivity to about $\pm 5 \times 10^{-4}$, while the broadening of the $\mathrm{InGaAs} / \mathrm{InP}$ interfaces allows us to estimate the $z$ resolution to about $9 \mathrm{~nm}$.

Additional structural information can be extracted from the phase map. Indeed, the diffraction patterns shown in Fig. 4 present strong indications of the presence of crystal plane tilts, corresponding to the rotation of the planes perpendicular to the 004 Bragg vector. The 3D maps of the two possible tilts can be extracted from the partial derivatives of $\phi_{004}(\mathbf{r})$ with respect to $x$ or $y$ [13]. The rotation of the planes around the axis perpendicular to the $(x, z)$ plane, i.e., the $y$ axis, is given by [see Eq. (6) below]

$$
\delta_{y}(\mathbf{r})=\sin ^{-1}\left(\left|\mathbf{G}_{004}\right|^{-1} \times \frac{\partial \phi_{004}}{\partial x}\right),
$$

while the rotation around the $x$ axis, $\delta_{x}(\mathbf{r})$, is obtained by exchanging $x$ and $y$ in the above expression.

Those 3D tilt maps are shown in Fig. 6, as 2D cross sections in the $(x, z)$ plane taken along $y$. As foreseen with the analysis of the data set, their behavior is not homogeneous but presents spatial variations. For both $\delta_{x}$ and $\delta_{y}$, we observe that the volume can be divided into two regions. On the larger $x$ side, the tilts are rather constant, taken as an orientation reference and therefore being equal to 0 on average. On the other region corresponding to the lower values of $x$, the $\delta_{x}$ tilt value increases up to $\approx 0.02^{\circ}$. For $\delta_{y}$, the tilt is first decreasing down to $\approx-0.02^{\circ}$ before increasing up to $\approx 0.02^{\circ}$, from the right to the left of the retrieved areas. Those behaviors are in agreement with the angular motion of the Bragg peaks observed in the data set, as detailed below. 

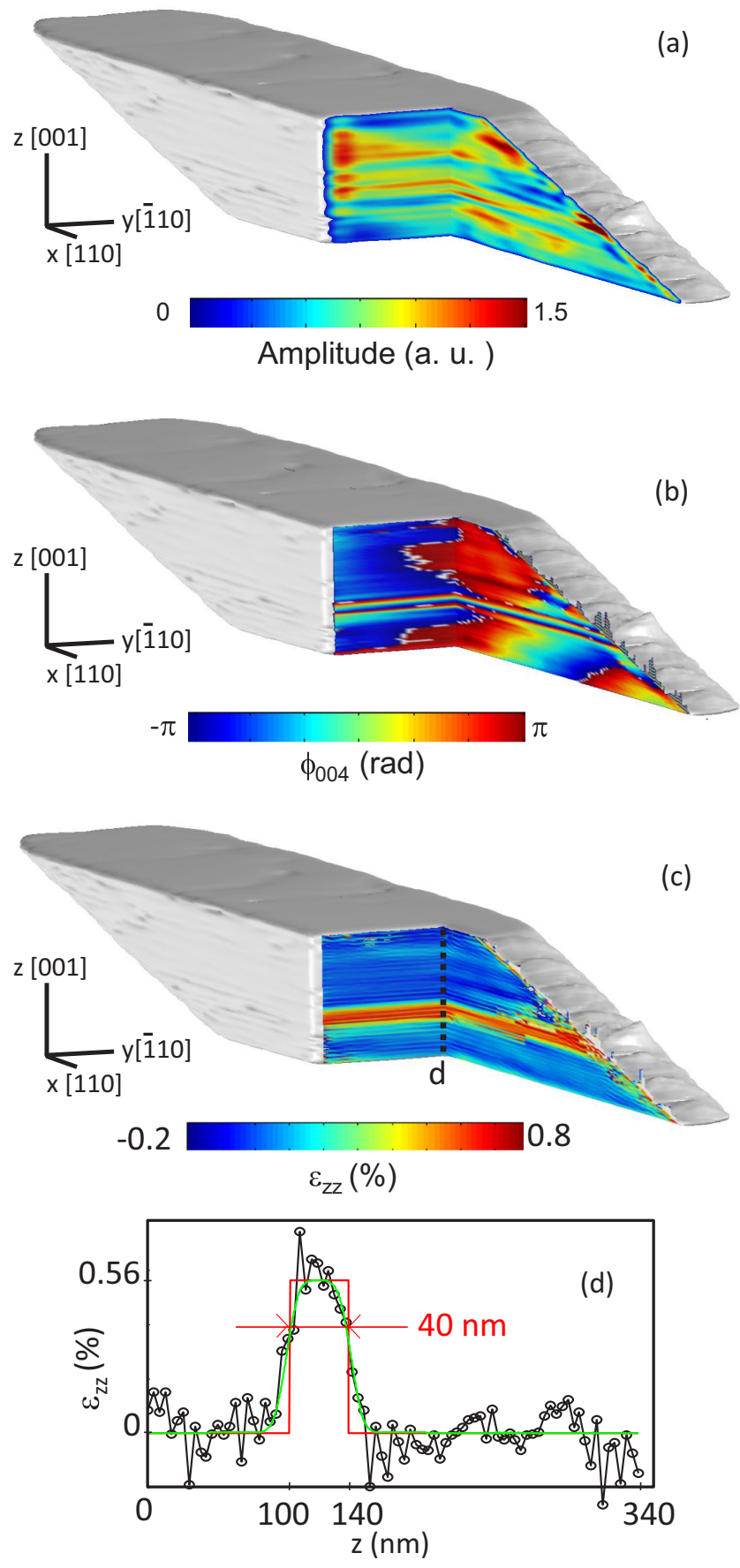

FIG. 5. (Color online) 3D quantities retrieved from the Bragg ptychography inversion. (a) 3D amplitude and (b) 3D phase resulting directly from the inversion of the Bragg ptychography data set. (c) 3D $\epsilon_{z z}$ strain component. (d) $\epsilon_{z z}$ 1D profile taken along the black line of (c). The red curve corresponds to the ideal profile while the green one results from the convolution of the ideal profile with a Gaussian resolution function of variance of $9 \mathrm{~nm}$. In (a)-(c), the shown volume corresponds to the part of the sample illuminated during the scan and the length of the black lines is $200 \mathrm{~nm}$.

\section{DISCUSSION}

Our Bragg ptychography reconstruction presents structural details in full agreement with the nominal values of the InP nanostructured stack. In particular, the InGaAs embedded

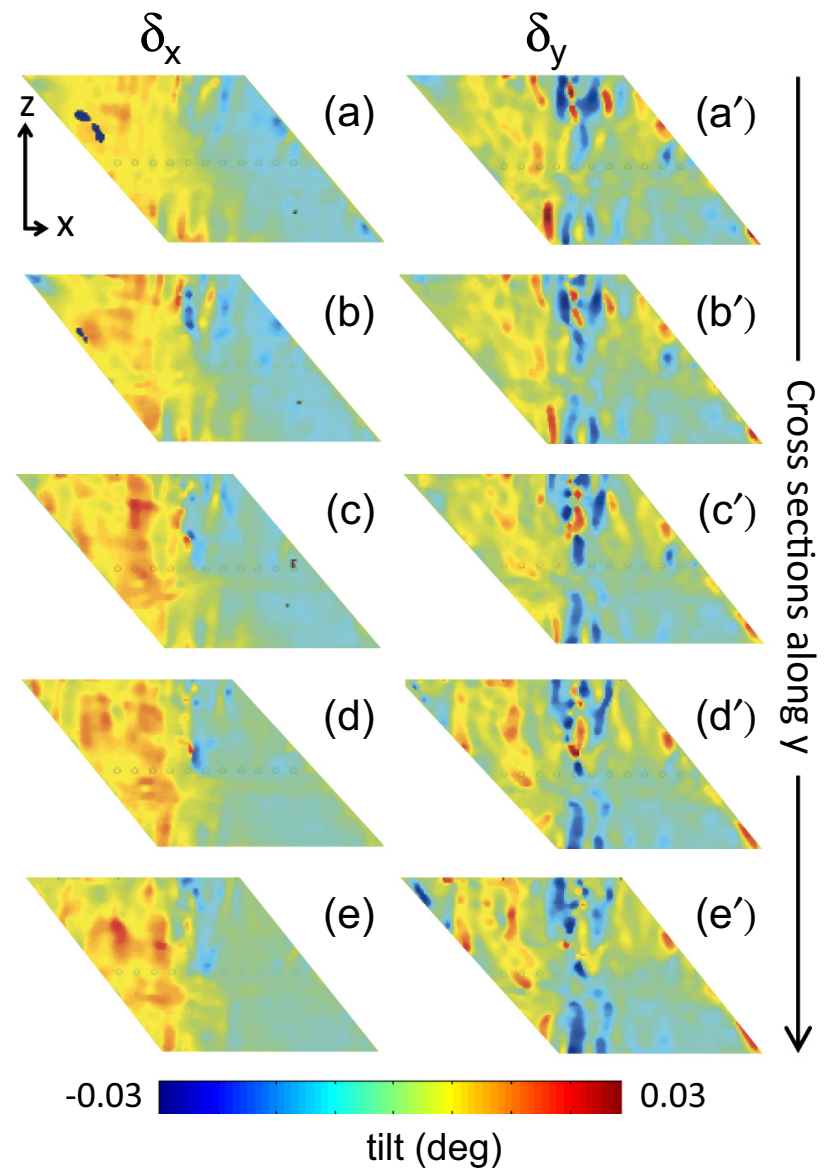

FIG. 6. (Color online) 3D tilt maps extracted from $\phi_{004}(\mathbf{r})$ using Eq. (4). Rotation of the 001 crystalline planes (a)-(e) around the $x$ axis and $\left(\mathrm{a}^{\prime}\right)-\left(\mathrm{e}^{\prime}\right)$ around the $y$ axis. The $2 \mathrm{D}$ cross sections are taken along $y$ in the $(x, z)$ planes, spaced every $140 \mathrm{~nm}$ (see Fig. 5). The colored angular scale is given at the bottom. The black arrows are $200 \mathrm{~nm}$ long.

layer has been retrieved with a good accuracy for position, thickness, and lattice mismatch values. However, the presence of tilts in the retrieved sample image was not expected and requires a detailed discussion. In the following, we address the following three questions: (i) Are the data of good quality? (ii) How does a spatially localized crystalline tilt modify the diffraction pattern? (iii) How do our results compare to the electron microscopy observations?

\section{A. Quality of the Bragg ptychography data set}

The success of the Bragg ptychography reconstruction relies on the quality of the data set. The usual difficulties that can be encountered during an experiment involving a nanofocused coherent x-ray beam include setup instabilities and sample motion misalignment. These issues were carefully addressed during the acquisition of the ptychography data, as discussed now.

Setup instabilities may be of two kinds: short-time-scale vibrations and long-time-scale drifts. The stability of the setup on short time scales has been carefully checked and is evidenced in the acquisitions shown in Fig. 7. The upper part 

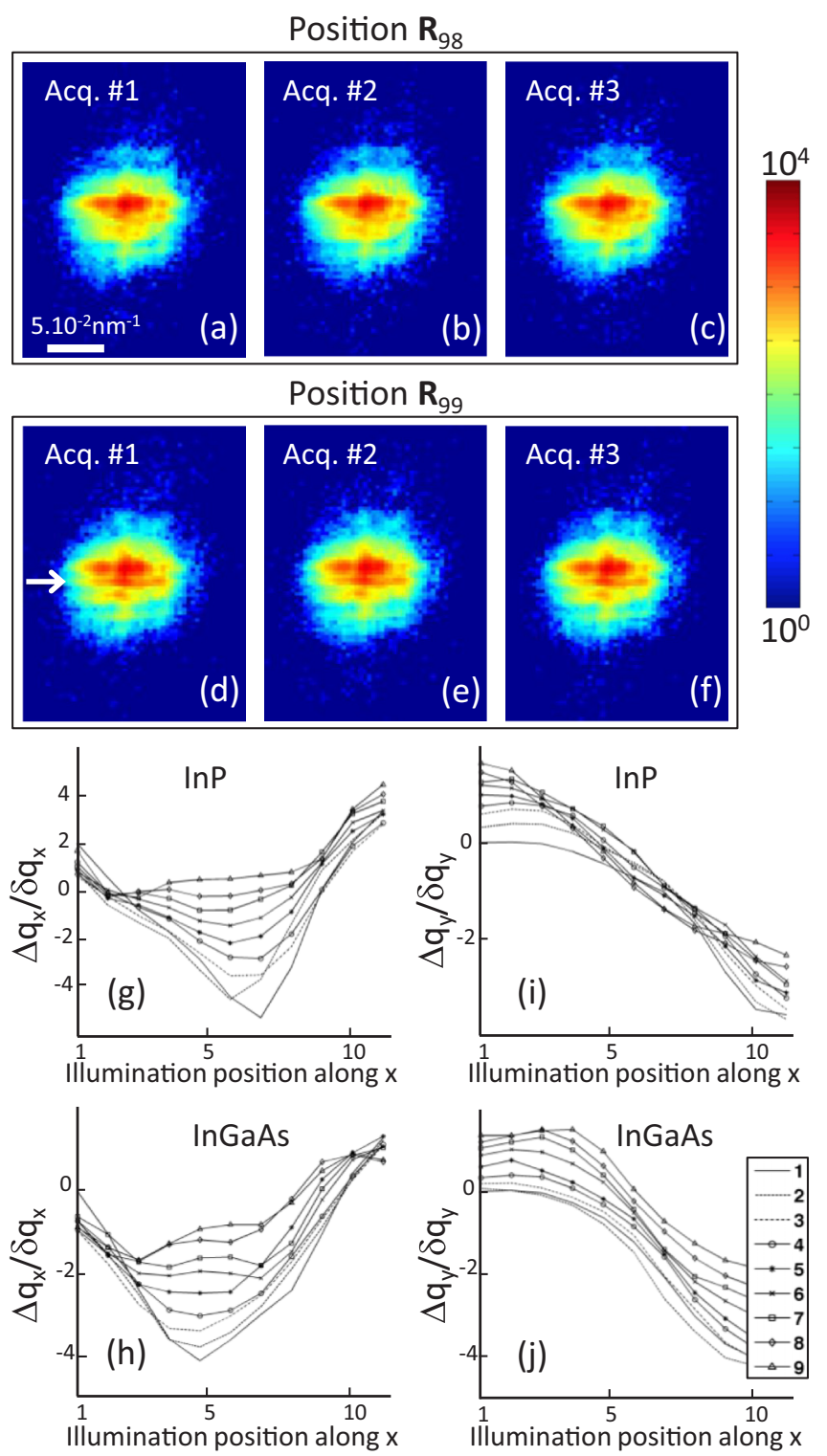

FIG. 7. (Color online) Evaluation of the x-ray data quality. (a)-(c) Three successive acquisitions of the Bragg coherent diffraction signal performed at the same beam-to-sample position, namely $\mathbf{R}_{98}$. These diffraction patterns differ only at pixels with low counts, as a result of photon shot noise statistics. (d)-(f) Same as (a)-(c), obtained at the next beam-to-sample position $\left(\mathbf{R}_{99}\right)$. Clear differences are evidenced at higher intensity levels between the two different positions, as pointed out by the white arrow in (d). (g)-(j) Motions of the Bragg peaks given in pixel numbers with respect to the position of the peak position at the first beam-to-sample position. (g), (h) Motion along the $q_{x}$ direction for the InP and InGaAs peaks, respectively. (i), (j) Same as (g), (h) along the $q_{y}$ direction. The horizontal axis corresponds to the illumination position along $x$ while the different curves are produced when varying the illumination position along $y$, following the legend in (j).

of the figure [from (a) to (c)] corresponds to three successive acquisitions performed at the very same angular and beamto-sample positions. The only visible differences are limited to the low count intensity pixels, as expected from the photon shot noise process. The next three acquisitions [from (d) to (f)],

which are again very similar one to the other, correspond to the next beam-to-sample position. They have been measured right after the three ones above. Clear differences between these two data groups are observed, even in the high count intensity pixels (see the thickness fringes [white arrow on (d)]). The comparison of these two data sets brings two major pieces of information. (i) The likelihood between the diffraction patterns obtained at the same position confirms the stability of the setup on short time scales (i.e., a few seconds). (ii) The important discrepancies observed between diffraction patterns obtained at nearby beam-to-sample positions indicate the existence of structural fluctuations within the sample.

Long-time-scale positional drifts are more difficult to evidence in the case of an extended sample. They can be related either to temperature fluctuations or to some mechanical shifts. To avoid temperature-dependent drifts, the design of the ID13 experimental hutch includes a thermal isolation. Furthermore we have regularly checked the absence of drift, during various experimental campaigns, using finite-sized test samples, like the one used in Ref. [10], for which a successful data set was obtained without realigning the sample positions during the acquisition. A good indication of the absence of drift in our data set is given by the plots shown in Figs. 7(g)-7(i), where the motions of the InP and InGaAs Bragg peaks are monitored as a function of the illumination position. We observe that both peaks, measured at quite different times during the ptychography acquisition, are following the same trends. This is a strong indication of consistency in the data set. Interestingly the motions of the InP and InGaAs peaks are not of the same amplitude [see, e.g., Figs. 7(g), 7(h)], which invalidates the existence of a mechanical problem such as a global misorientation of the sample at some specific position along the piezo-stage translations. Data consistency is further confirmed by the smoothness of the build-up 3D diffraction patterns as a function of the beam-to-sample positions (Fig. 4). These observations argue in favor of the stability of the setup on time scales longer than the total acquisition time.

Another interesting question relates to the impact of any sample-to-beam misalignment onto the data quality. The ptychography scan requires that the beam illuminates the sample at a position that remains the same all along the rocking curve scan. We already saw in Sec. III that the shrinkage of the footprint during the rocking curve scan can be neglected. Moreover, the center of rotation of the sample has to coincide with the beam focus, a condition which is never fully verified in practice. Along the beam incidence direction, one can tolerate some positioning errors as the depth of field of the $\mathrm{X}$-ray beam is a few hundreds of micrometers long [26]. Note that this geometrical error is not producing a change of the illumination area as a function of the angle of incidence. The situation is slightly different for the alignment in the plane perpendicular to the beam, where a shift $l_{c}$ of the sample center of rotation with respect to the beam center is leading to the illumination of different volumes during the rocking curve scan. One can estimate the corresponding motion, $\Delta_{x}$, of the illumination onto the sample surface during an angular scan of amplitude $\Delta \theta$, given by $\Delta x=l_{c} \Delta \theta / \tan \theta_{B}$. For our setup, the $l_{c}$ shift is minimized with the alignment procedure that relies on the optical microscope specifications regarding the 
depth of field. Typically, $l_{c} \leqslant 500 \mathrm{~nm}$ results in $\Delta x \leqslant 15 \mathrm{~nm}$, i.e., a spatial shift comparable to one pixel along the $x$ axis (5\% of the footprint). Here again, the consistency exhibited in the data set argues in favor of negligible misalignment issues.

Finally, another source of inconsistency in the data set results from the uncertainty of the piezo stage. The 3D data being produced by the stacking of $2 \mathrm{D}$ patterns acquired when addressing sequentially the beam-to-sample positions, we expect this effect to be averaged along the 180 patterns used to describe the full rocking curve. This question could be however addressed with the newly developed formalism presented in Ref. [25].

\section{B. Introducing a numerical model accounting for local crystalline plane tilts}

The discrepancies observed between the defect-free model and the experimental data (Figs. 3 and 4) invoked the presence of spatially localized rotations of the crystalline planes, further evidenced in Fig. 6. We note that the tilt distributions as revealed by the ptychography $3 \mathrm{D}$ reconstruction (Fig. 6) follow fully the motions of the Bragg peaks, as monitored in Figs. 7(g)-7(j). To go further in the analysis, we describe here the model to account for those tilted structures and compare the produced diffraction pattern to the experimental data.

The tilt $\delta$ is a rotation of the 001 planes around an axis being normal to the rotation plane, referred to as the tilt axis [Fig. 8(a)]. Consequently, with respect to a reference crystal orientation, a tilted crystal induces the rotation of all associated Bragg vectors. Note that if the tilt axis and the chosen Bragg vector are collinear, the rotation does not affect the Bragg vector position. For the other Bragg vectors, the complex-valued electron density has to be modified. The tilt expresses itself as an additional displacement field component $u_{t}$. One can easily be convinced that $u_{t}$ is varying linearly with the spatial coordinates perpendicular to the tilt axis, i.e., $x$ and $z$ for the 2D case depicted in Fig. 8(b). More precisely, the displacement field component associated with the 001 direction is given, in the tilted region, by

$$
u_{t, 001}(x, z, \delta)=x \sin \delta-z(1-\cos \delta),
$$

from which we can derive the phase offset $\phi_{t, 004}$ associated with the $\mathbf{G}_{004}$ Bragg vector

$$
\phi_{t, 004}(x, z, \delta)=\left|\mathbf{G}_{004}\right| \times[x \sin \delta-z(1-\cos \delta)] .
$$

It is worth noting that for $\delta \ll 1$, the above expression can be approximated to

$$
\phi_{t, 004}(x, z, \delta)=\left|\mathbf{G}_{004}\right| \times\left(x \delta-\frac{1}{2} z \delta^{2}\right),
$$

where the second term in the parentheses is negligible with respect to the first one, as long as $x$ and $z$ are spanning comparable distances. Hence, a small angular tilt affects mostly the electron density phase along the directions perpendicular to the chosen Bragg vector.

The above model [Eq. (6)] was used to introduce a local tilt in the numerical model built on the nominal structural parameters of the InP nanostructured layer [Figs. 3(d), 3(e) and $\left.9(\mathrm{a}), 9\left(\mathrm{a}^{\prime}\right)\right]$. Several configurations were tested, where one (a)

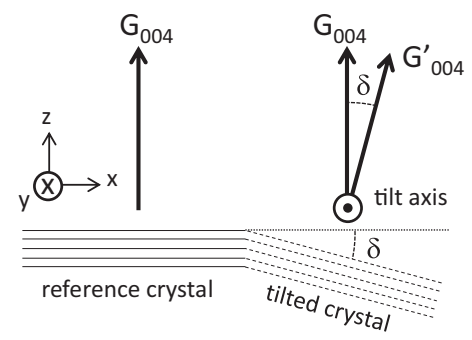

(b)

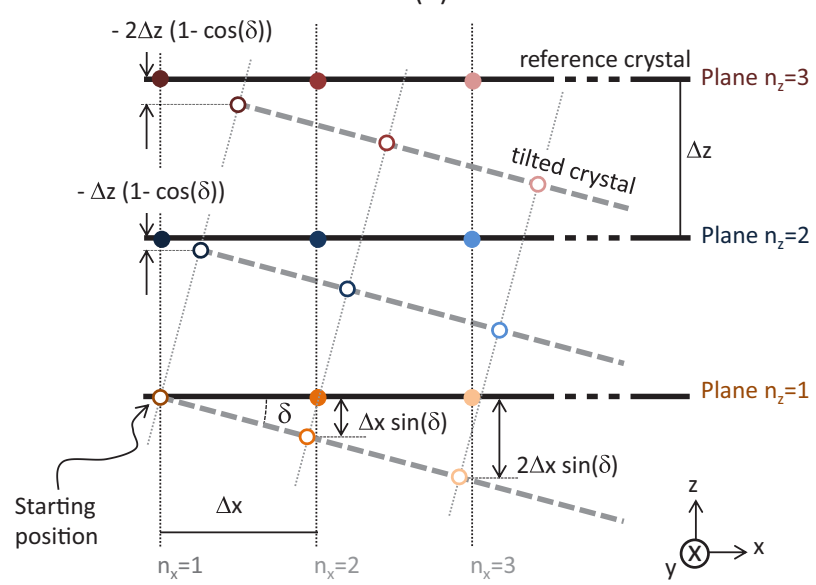

FIG. 8. (Color online) Introduction of a tilted crystal. (a) A crystal, whose diffracting planes are tilted by an angle $\delta$ about a tilt axis perpendicular to the Bragg vector induces a corresponding rotation of the Bragg vector. (b) The rotation of the tilted crystal leads to the introduction of a displacement field, that expresses the difference between the plane positions in the tilted crystal with respect to the plane positions in the reference crystal, projected along the Bragg vector direction. Some typical values of the displacement field are given in the figure.

or two tilts were introduced, with different values for $\delta$, tilt width along $x$, and vertical extension limited to some or all the InP and InGaAs layers. The diffraction pattern presented in Figs. 9(b), 9(b') was performed with the introduction of two tilts separated by $400 \mathrm{~nm}$ along $x$, affecting equally the whole sample stack, with rotation angles $\delta= \pm 0.02^{\circ}$ around the $\mathbf{y}$ axis. The differences with the nontilted case is obvious: distortions and extensions of the Bragg peaks, splitting of the InGaAs peak along $\mathbf{q}_{x}$. The obtained qualitative agreement between the simulated data and the experimental data [see arrows in Figs. $9\left(b^{\prime}\right)$ and $9\left(c^{\prime}\right)$ ] was achieved although we restricted the model to a minimum number of fitting parameters, on purpose. The retrieved phase map, shown in Fig. 9(c) on a wrapped representation, exhibits as well the specific behavior of a locally tilted crystal according to our calculation. On the whole, this model, which reproduces fairly the experimental observations, demonstrates the compatibility between this hypothesis and our Bragg ptychography reconstruction. 
(a)
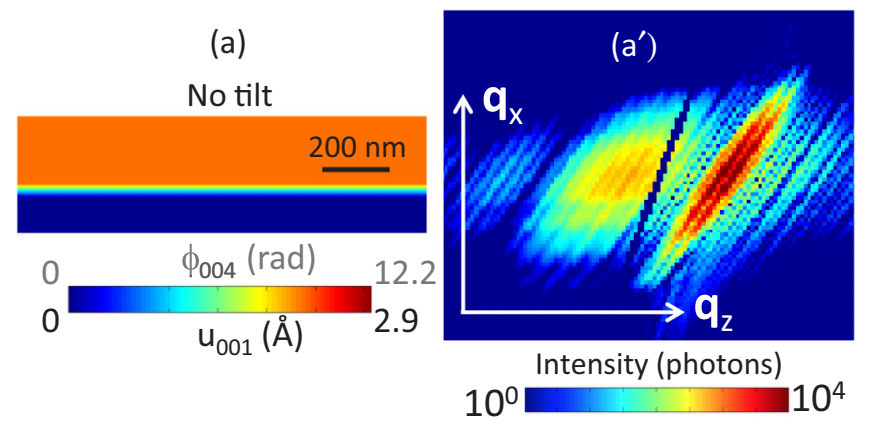

(b)

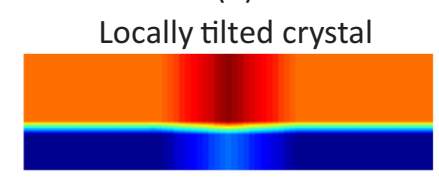

(c)
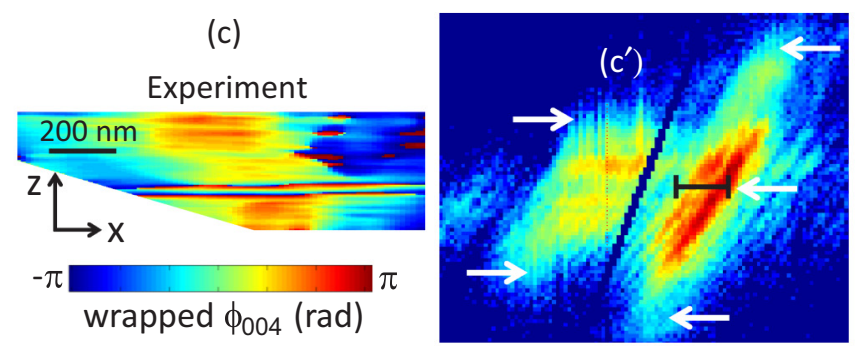

FIG. 9. (Color online) Numerical simulation with a local crystalline tilt. (a) and ( $\left.a^{\prime}\right)$ Numerical model built on structural parameters corresponding to the nominal values of the InP layered structure (see Figs. 3 and 4). (b) and ( $b^{\prime}$ ) Same as (a), with the additional introduction of two tilts $\left(\delta= \pm 0.02^{\circ}\right)$ separated by $400 \mathrm{~nm}$. In (a) and (b) the top maps present the $\phi_{004}(\mathbf{r})$ and $u_{001}(\mathbf{r})$ distributions while in $\left(\mathrm{b}^{\prime}\right)$ and $\left(\mathrm{c}^{\prime}\right)$ the intensity patterns are $2 \mathrm{D}$ cross sections extracted from the 3D intensity simulations. (c) and ( $\left.\mathrm{c}^{\prime}\right)$ Experimental results. (c) Retrieved phase shown on a wrapped representation and $\left(\mathrm{c}^{\prime}\right)$ 2D cross-section extracted from the experimental intensity patterns [identical to Fig. 4(d)]. In (b'), the arrows are pointing to the onsets of the crystalline plane rotations, which produce intensity distortions similar to the experimental ones, marked similarly in $\left(\mathrm{c}^{\prime}\right)$. The length of the axes corresponds to $5 \times 10^{-2}$ and $5 \times 10^{-1} \mathrm{~nm}^{-1}$ for the $\mathbf{q}_{x}$ and $\mathbf{q}_{z}$ directions, respectively.

\section{Comparison with transmission electron microscopy results}

At first sight, the results obtained with STEM and Bragg ptychography are apparently in contradiction. Apart from the chemical fluctuations observed at the InP/Si interface, none of the structural inhomogeneities observed during the ptychography experiments (data and reconstruction) were observed with STEM. However, if one wants to compare these two microscopy approaches, it is of major importance to have in mind their respective specificities. STEM provides crystal images with an extremely high spatial resolution, restricted to a 2D spatial field of view of about $0.1 \mu \mathrm{m}$. The sensitivity of the setup was estimated from the measurements themselves (Fig. 10) with a geometrical phase analysis [28] allowing us to extract the $r_{x z}$ rotation (around the $y$ axis) and the
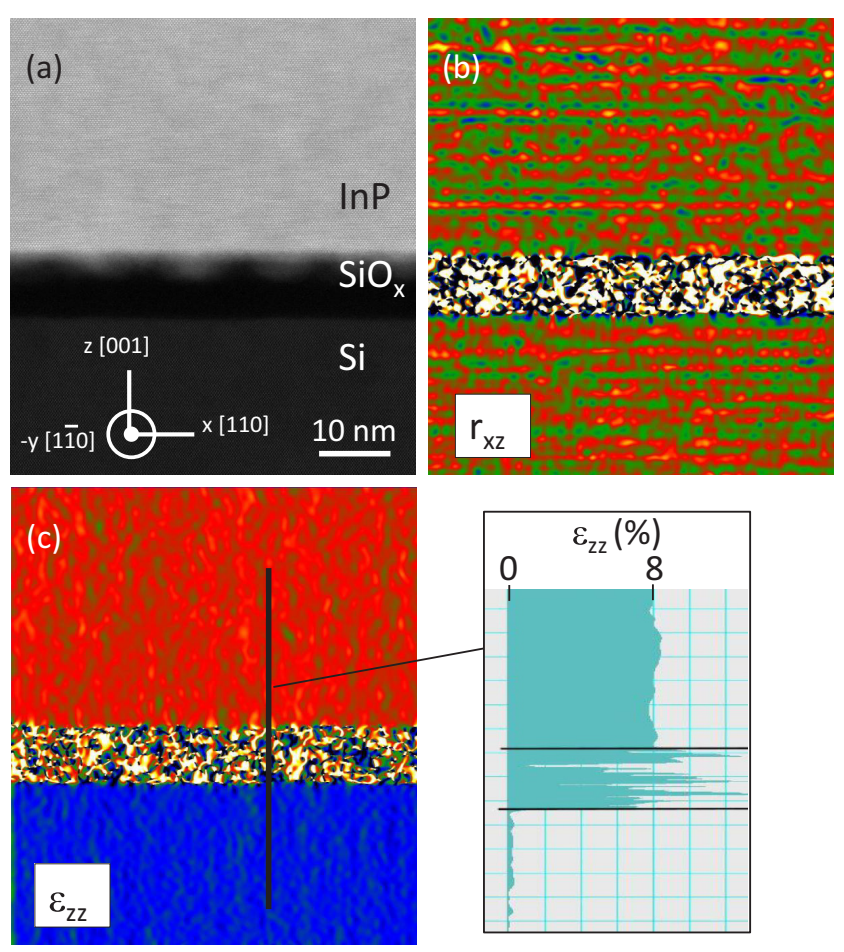

FIG. 10. (Color online) Quantifying STEM sensitivity. (a) HAADF mode view of the $\mathrm{InP} / \mathrm{Si}$ interface. (b) Rotation $r_{x z}$ and (c) strain $\epsilon_{z z}$ maps obtained from (a) using a geometrical phase analysis. The color scale in (b) goes from $-0.5^{\circ}$ to $+0.5^{\circ}$, while in the inset of (c), the 1D cross section of $\epsilon_{z z}$ along $z$ exhibits fluctuations of about $\pm 0.3 \%$.

$\epsilon_{z z}$ strain (along the $z$ [001] direction). The presence of fluctuations on the images shown in Figs. 10(b) and 10(c) allows us to quantify the sensitivity to crystalline rotations to about $0.5^{\circ}$ and the accuracy on $\epsilon_{z z}$ to about \pm 0.003 . On the contrary, our 3D Bragg ptychography reconstruction is extremely sensitive to the crystalline distortions [a lower bound of about $0.005^{\circ}$ is estimated from Fig. 4(d)] and carries long-length-scale information over a large volume (here about $\left.2 \times 0.4 \times 0.34 \mu \mathrm{m}^{3}\right)$. Similar difficulties in comparing Bragg ptychography and electron microscopy results have been reported $[10,11]$ and more generally, due to the specificities of the 3D Bragg ptychography images, we stipulate that none of the existing electron or x-ray diffraction based microscopy approaches can provide crystalline images truly relevant for comparison. This highlights the specific role to be played by 3D Bragg ptychography in crystal microscopy.

Finally, we would like to underline that understanding the origin of the observed features is out of reach of this work. Indeed, it is possible to address this question with additional analysis tools, such as the finite-element model, in order to calculate the displacement field according to the crystal elastic constant and test different structural conditions (nonhomogeneous chemical composition, nonhomogeneous pressure during bonding, presence of dislocations, etc.). This should undoubtedly result in the finding of an adequate scenario able at explaining the observed structural features. However, a deep understanding of the origin of the local tilts 
in the crystal requires at the end validating the proposed model during dedicated experimental campaigns. This is beyond the scope of the present article.

\section{CONCLUSION}

In conclusion, we have demonstrated the possibility of imaging in 3D the crystalline properties of an extended nanostructured layer without sample preparation. The shown results emphasize the specificities of Bragg ptychography, which holds exciting perspectives for the investigation of complex nanostructured crystals, arising either from nanotechnology or produced by living species.

\section{ACKNOWLEDGMENTS}

We are grateful to S. Lazarev and D. Grigoriev, who are warmly acknowledged for their help during the synchrotron experiment, and to T. Baumbach for fruitful discussions. O. Mauguin is acknowledged for her help on the sample characterization. P. Ferrand is warmly acknowledged for fruitful discussions and his careful reading of the manuscript. The ESRF is acknowledged for allowance of beamtime. The French ANR (Program No. ANR-10-EQPX-50) is acknowledged for support regarding the use of the dual-beam FEI SCIOS system. A.P.'s Ph.D. thesis is supported by the Erasmus Mundus Doctorate Program Europhotonics (Grant No. 159224-1-20091-FR-ERAMUNDUS-EMJD).
[1] M. J. Suess, R. Geiger, R. A. Minamisawa, G. Schiefler, J. Frigerio, D. Chrastina, G. Isella, R. Spolenak, J. Faist, and H. Sigg, Analysis of enhanced light emission from highly strained germanium microbridges, Nat. Photonics 7, 466 (2013).

[2] G. Catalan, A. Lubk, A. H. G. Vlooswijk, E. Snoeck, C. Magen, A. Janssens, G. Rispens, G. Rijnders, D. H. A. Blank, and B. Noheda, Flexoelectric rotation of polarization in ferroelectric thin films, Nat. Mater. 10, 963 (2011).

[3] B. Bayerlein, P. Zaslansky, Y. Dauphin, A. Rack, P. Fratzl, and I. Zlotnikov, Self-similar mesostructure evolution of the growing mollusc shell reminiscent of thermodynamically driven grain growth, Nat. Mater. 13, 1102 (2014).

[4] M. Hÿtch, F. Houdellier, F. Hüe, and E. Snoeck, Nanoscale holographic interferometry for strain measurements in electronic devices, Nature (London) 453, 1086 (2008).

[5] J. Stangl, C. Mocuta, V. Chamard, and D. Carbone, Nanobeam $X$-Ray Scattering: Probing Matter at the Nanoscale (Wiley, Weinheim, Germany, 2013).

[6] M. A. Pfeifer, G. J. Williams, I. A. Vartanyants, R. Harder, and I. K. Robinson, Three-dimensional mapping of a deformation field inside a nanocrystal, Nature (London) 442, 63 (2006).

[7] V. Chamard, J. Stangl, G. Carbone, A. Diaz, G. Chen, C. Alfonso, C. Mocuta, and T. H. Metzger, Three-Dimensional X-Ray Fourier Transform Holography: The Bragg Case, Phys. Rev. Lett. 104, 165501 (2010).

[8] P. Godard, G. Carbone, M. Allain, F. Mastropietro, G. Chen, L. Capello, A. Diaz, T. H. Metzger, J. Stangl, and V. Chamard, Three-dimensional high-resolution quantitative microscopy of extended crystals, Nat. Commun. 2, 568 (2011).

[9] F. Berenguer, P. Godard, M. Allain, J.-M. Belloir, A. Talneau, S. Ravy, and V. Chamard, X-ray lensless microscopy from undersampled diffraction intensities, Phys. Rev. B 88, 144101 (2013).

[10] V. Chamard, M. Allain, P. Godard, A. Talneau, G. Patriarche, and M. Burghammer, Strain in a silicon-on-insulator nanostructure revealed by 3D x-ray Bragg ptychography, Sci. Rep. 5, 9827 (2015).

[11] S. O. Hruszkewycz, M. V. Holt, C. E. Murray, J. Bruley, J. Holt, A. Tripathi, O. G. Shpyrko, I. McNulty, M. J. Highland, and P. H. Fuoss, Quantitative nanoscale imaging of lattice distortions in epitaxial semiconductor heterostructures using nanofocused x-ray Bragg projection ptychography, Nano Lett. 12, 5148 (2012).
[12] S. O. Hruszkewycz, M. J. Highland, M. V. Holt, D. Kim, C. M. Folkman, C. Thompson, A. Tripathi, G. B. Stephenson, S. Hong, and P. H. Fuoss, Imaging Local Polarization in Ferroelectric Thin Films by Coherent X-Ray Bragg Projection Ptychography, Phys. Rev. Lett. 110, 177601 (2013).

[13] M. V. Holt, S. O. Hruszkewycz, C. E. Murray, J. R. Holt, D. M. Paskiewicz, and P. H. Fuoss, Strain Imaging of Nanoscale Semiconductor Heterostructures with X-Ray Bragg Projection Ptychography, Phys. Rev. Lett. 112, 165502 (2014).

[14] Y. Takahashi, A. Suzuki, S. Furutaku, K. Yamauchi, Y. Kohmura, and T. Ishikawa, Bragg x-ray ptychography of a silicon crystal: Visualization of the dislocation strain field and the production of a vortex beam, Phys. Rev. B 87, 121201 (2013).

[15] K. Tanabe, K. Watanabe, and Y. Arakawa, III-V/Si hybrid photonic devices by direct fusion bonding, Sci. Rep. 2, 349 (2012).

[16] A. Itawi, K. Pantzas, I. Sagnes, G. Patriarche, and A. Talneau, Void-free direct bonding of InP to Si: Advantages of low $\mathrm{H}$ content and ozone activation, J. Vac. Sci. Technol. B 32, 021201 (2014).

[17] A. Talneau, C. Roblin, A. Itawi, O. Mauguin, L. Largeau, G. Beaudoin, I. Sagnes, G. Patriarche, C. Pang, and H. Benisty, Atomic-plane-thick reconstruction across the interface during heteroepitaxial bonding of InP-Clad quantum wells on silicon, Appl. Phys. Lett. 102, 212101 (2013).

[18] J. Rodenburg and R. Bates, The theory of super-resolution electron microscopy via Wigner-distribution deconvolution, Philos. Trans. R. Soc. London A 339, 521 (1992).

[19] J. M. Rodenburg, A. C. Hurst, A. G. Cullis, B. R. Dobson, F. Pfeiffer, O. Bunk, C. David, K. Jefimovs, and I. Johnson, Hard-X-Ray Lensless Imaging of Extended Objects, Phys. Rev. Lett. 98, 034801 (2007).

[20] P. Thibault, M. Dierolf, A. Menzel, O. Bunk, C. David, and F. Pfeiffer, High-resolution scanning x-ray diffraction microscopy, Science 321, 379 (2008).

[21] M. Dierolf, A. Menzel, P. Thibault, P. Schneider, C. Kewish, R. Wepf, O. Bunk, and F. Pfeiffer, Ptychographic X-ray computed tomography at the nanoscale, Nature (London) 467, 436 (2010).

[22] H. M. Quiney, A. G. Peele, Z. Cai, D. Paterson, and K. A. Nugent, Diffractive imaging of highly focused x-ray field, Nat. Phys. 2, 101 (2006). 
[23] C. Ponchut, J. Clément, J.-M. Rigal, E. Papillon, J. Vallerga, D. LaMarra, and B. Mikulec, Photon-counting x-ray imaging at kilohertz frame rates, Nucl. Instrum. Methods A 576, 109 (2007).

[24] S. Takagi, A dynamical theory of diffraction for a distorted crystal, J. Phys. Soc. Jpn. 26, 1239 (1969).

[25] S. O. Hruszkewycz, M. Allain, M. V. Holt, C. E. Murray, J. R. Holt, P. H. Fuoss, and V. Chamard, High resolution three dimensional structural microscopy by single angle Bragg ptychography, arXiv:1506.01262.
[26] F. Mastropietro, D. Carbone, A. Diaz, J. Eymery, A. Sentenac, T. H. Metzger, V. Chamard, and V. Favre-Nicolin, Coherent X-ray wavefront reconstruction of a partially illuminated Fresnel zone plate, Opt. Express 19, 19223 (2011).

[27] P. Godard, M. Allain, V. Chamard, and J. Rodenburg, Noise models for low counting rate coherent diffraction imaging, Opt. Express 20, 25914 (2012).

[28] K. Ishizuka, A Dedicated Site for Quantitative Electron Microscopy, date of access 1 June 2015, http://www.hremresearch.com. 